\title{
Modeling Latency in a Network Distribution
}

\author{
Rohitha Goonatilake ${ }^{1} \&$ Rafic A. Bachnak ${ }^{1}$ \\ ${ }^{1}$ Department of Engineering, Mathematics, and Physics, Texas A\&M International University, Laredo, USA \\ Correspondence: Rohitha Goonatilake, Department of Engineering, Mathematics, and Physics, Texas A\&M \\ International University, 5201 University Boulevard, Laredo, USA. E-mail: harag@tamiu.edu
}

Received: July 31, 2012 Accepted: August 15, 2012 Online Published: August 28, 2012

doi:10.5539/nct.v1n2p1 URL: http://dx.doi.org/10.5539/nct.v1n2p1

\begin{abstract}
Network latency causes a delay in transmitting a message from one location to another. This can be attributed to several other factors, such as network congestion, network traffic, and computer storage capacities. Of course, the distance between two locations is the main factor that contributes to the delay. Since transmission between two cities will not be a straight path, latency is subject to detour and can be a factor of any deviation between these cities. These factors, along with a loss of the data and energy aspects of the transmission, will be investigated as this paper attempts to summarize latency estimation using regression and numerical models. Path prediction can be done up to a number of transmission towers or satellites between two cities. Latency estimation to locate either the client, client server, or host will be analyzed using a liner regression model leading to the same numerical model. Reliability analysis stemming from latency will be done at the end of this article.
\end{abstract}

Keywords: latency, network, communication, distance, reliability, wireless, and packets

\section{Preliminaries}

The term latency refers to the time elapsed between the sending of a message to a router and the return of that message. That is, latency in a network is a term of how much time it takes for a packet of data to get from one designated point to another. This delay occurs when sending information across the network. A network is two or more computers which are connected to share information, software, data, and other activities. Latency can be measured by sending a packet that is returned to the sender; the round-trip time is considered the latency in a network provider such as AT\&T. Madhyastha et al. (2006) indicated that detecting better latency detours is one of the several applications enabled by their approach using paths measured from PlanetLab nodes. Back in 1914, there was evidence of the abnormal propagation of sound waves in the atmosphere, which later turned out to be latency (Fujiwhara, 1916). Increases of network speed and of bandwidth, and the use of better equipment have been cited as better solutions to diminish network latency.

In organizing this article, first, the factors associated with latency and their effects will be briefly discussed. The effect of latency with respect to distance between two communication equipments will be investigated, thereafter. This will lead to finding the number of satellites and towers between them. Numerical methods play a pivotal role in latency modeling. The Newton forward-divided differences are used to calculate distances between two locations from their known latencies. Its implication on volatile market and wireless network will be briefly stated. Finally, the article is concluded by conducting reliability analysis to determine quality of the network as a result of network latency over a long period of time. 




Figure 1. Communication propagation in a network system

Contributions to network latency include many factors such as propagation, transmission, router, other processing mechanism, other computer, and storage delays. Some of these are briefly described below:

(1) Propagation: The time it takes for a packet to travel between places at the speed of light.

(2) Transmission: The medium itself (optical fiber, wireless, or some other) introduces some delay. The size of the packet introduces delay in a round trip since a larger packet will take longer to receive and return than a small one.

(3) Router and other processing: Each gateway node takes time to examine and possibly changes the header in a packet (for example, changing the hop count in the time-to-live field).

(4) Other computer and storage delays: Within networks at each end of the journey, a packet may be subject to storage and hard disk access delays at intermediate devices such as switches and bridges. (In essence, however, this kind of latency is probably not considered).

(5) Other contributors such as contention and congestion can contribute to the network latency.

The total nodal delay in a network consists of processing, queuing, transmission, and propagation delays, $d_{\text {nodal }}=d_{\text {processing }}+d_{\text {queuing }}+d_{\text {transmission }}+d_{\text {propagation }}$. The propagation delay can be negligible amounting to a few microseconds. However, connection between two routers linking geostationary satellite link can perhaps contribute to major portion of the nodal delay. Estimating the propagation delay from the different users to the receiver and using off-line measurements have been previously done (Sinha, Raz, \& Choudhuri, 2006; Ström, Parkvall, \& Ottersten, 1994). Transmission delay varies from negligible to a rather significant amount, whereas, the processing remains small and corresponds to larger for a router that can forward packets (Kurose \& Ross, 2013).

Protocol and other technological implications could increase the network latency dramatically. However, researchers working on the designs of distributed databases (DDBs) ignore latency when performing file migration on LAN and incorporate them into the design phase (Johansson, 2000). In modeling latency, it is considered more important than the delays mainly caused by queuing. It is shown that the communication locality in the application improves the latency of direct networks, with a relative impact of increasing network load (Agarwal, 1991). The reduction of latency implementation of TCP (Transmission Control Protocol) on an ATM network such as header prediction and combination of checksum calculation with data copying can satisfactorily work to safeguard obvious private and public interests (Wolman, Voelker, \& Thekkath, 1994).

\section{Latency vs. Distance}

Latency depends on the distance between two locations. This aspect of latency will be further explored. Let $d_{1}$, $d_{2}$, and $d$ and $t_{1}, t_{2}$, and $t$ be the distances and times, respectively, taken to propagate a message from city 2 to the tower, from the tower to city 1 , and from city 1 to city 2 . The law of cosines states using $v$ as the rate of propagation of the wave,

$$
\begin{aligned}
d & =\frac{\sqrt{d_{1}^{2}+d_{2}^{2}-2 d_{1} d_{2} \cos \theta}}{}=\sqrt{\left(v t_{1}\right)^{2}+\left(v t_{2}\right)^{2}-2\left(v t_{1}\right)\left(v t_{2}\right) \cos \theta} \\
& =\quad v \sqrt{t_{1}^{2}+t_{2}^{2}-2 t_{1} t_{2} \cos \theta}
\end{aligned}
$$


Assuming $t_{1} \approx t_{2} \approx t$ and $\cos \theta \approx 0$, we have $d \approx \sqrt{2} v t$. If we introduce an additional tower and time taken from this tower to city 1 is $t_{3}$, then $d \approx \sqrt{(\sqrt{2} v t)^{2}+\left(v t_{3}\right)^{2}}=\sqrt{3} v t$ provided $t_{1} \approx t_{2} \approx t_{3} \approx t$. After continuing to add many towers, we have $d \approx \sqrt{n} v t$ for $n-1$ towers of transmission between city 1 and city 2 .

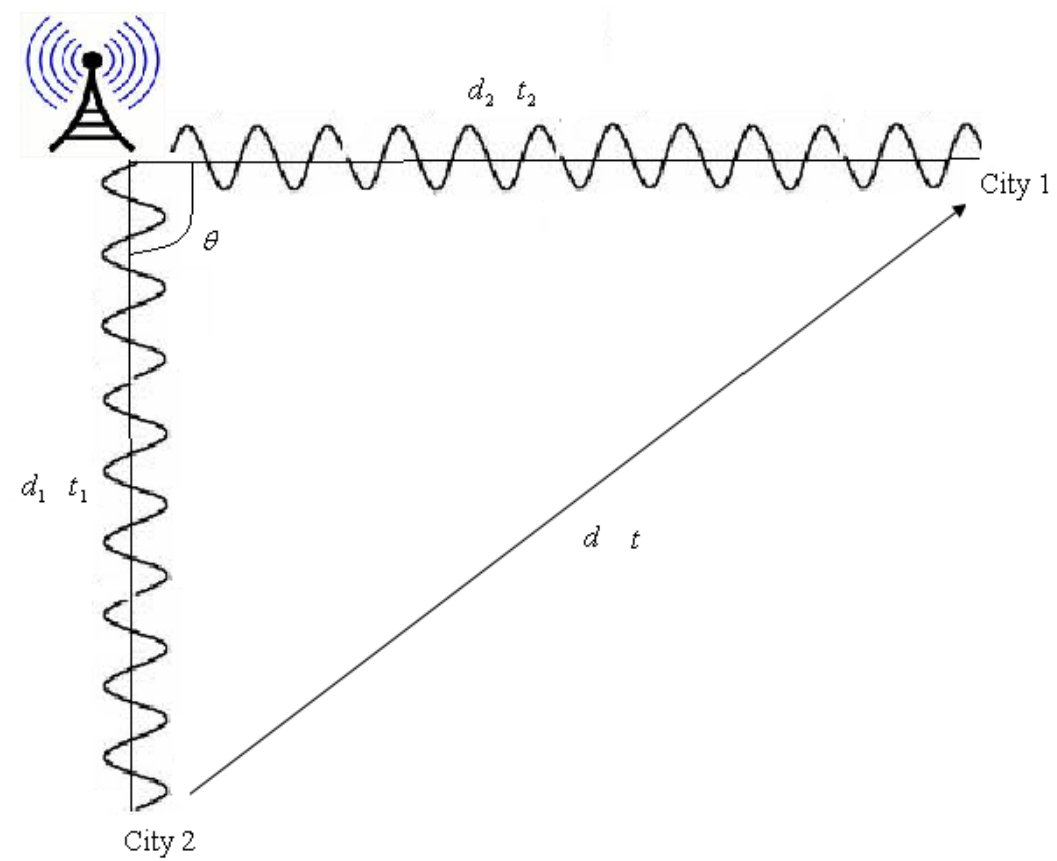

Figure 2. Transmission in a single tower serving two cities

If the linear regression model for time $(t)$ vs. distance $(d)$ is considered to be $\hat{t}=A+B d$ then $n t=A+B \times(\sqrt{n} v t)$.

This gives us $t=\frac{A}{n-B \sqrt{n} v} t=\frac{A}{n-B \sqrt{n v}} t=\frac{A}{n-B \sqrt{n} v} t=\frac{A}{n-B \sqrt{n} v} t=\frac{A}{n-B \sqrt{n} v} t=\frac{A}{n-B \sqrt{n} v}$. Furthermore,

$$
\begin{aligned}
d & =\sqrt{n} v t \\
& =\frac{\sqrt{n} v(A+B d)}{n} \\
& =\frac{v(A+B d)}{\sqrt{n}} \\
\sqrt{n} & =\frac{v}{d}(A+B d) \\
n & =\frac{v^{2}}{d^{2}}(A+B d)^{2} \\
n-1 & =\frac{v^{2}}{d^{2}}(A+B d)^{2}-1
\end{aligned}
$$
That is, there are $\frac{v^{2}}{d^{2}}(A+B d)^{2}-1$ transmission towers (rounded off to a nearest positive integer) in between
the two cities.

Communication between two locations can involve multiple transmission towers and satellites as depicted in Figure 3(a) and Figure 3(b). 


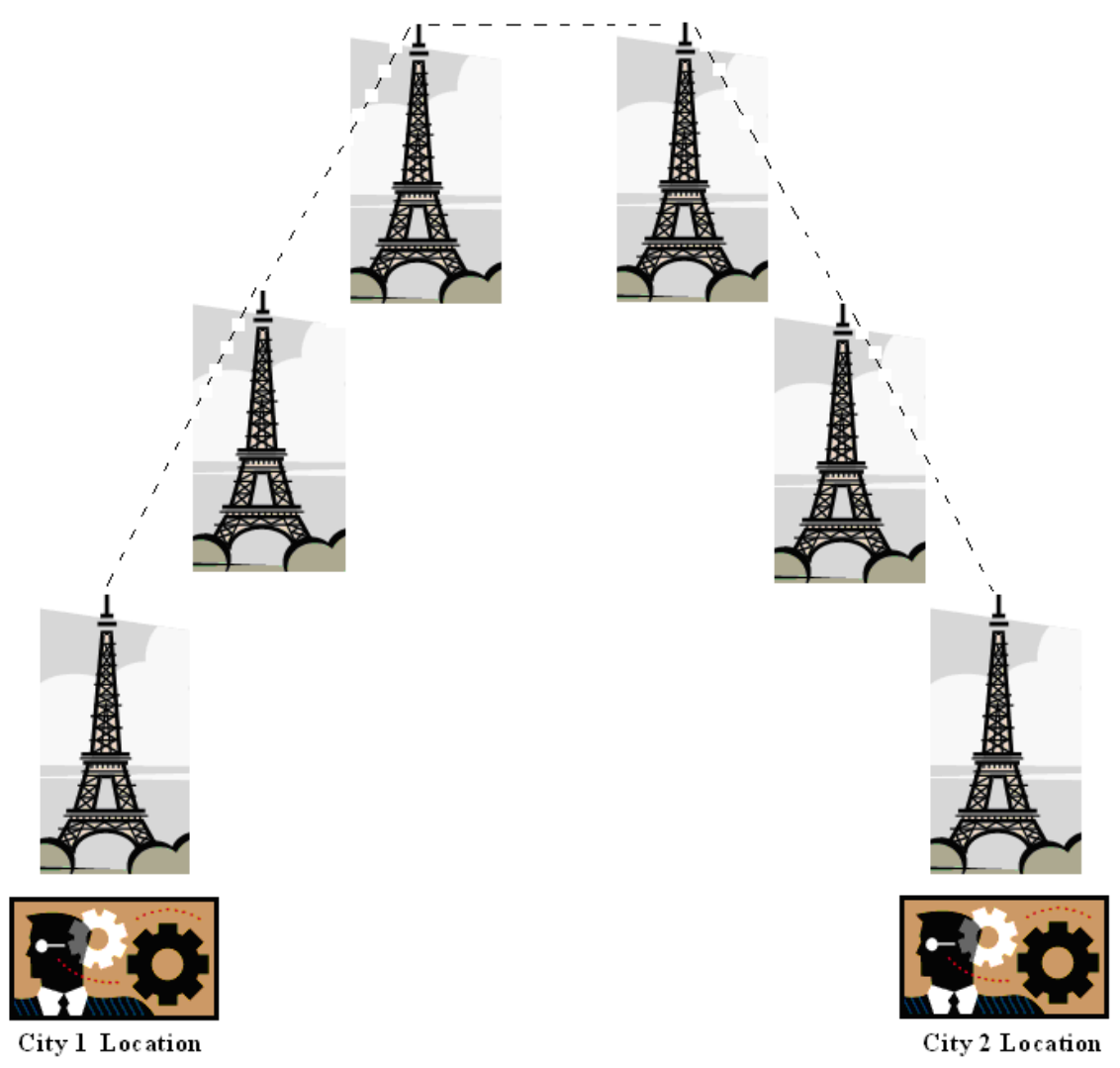

(a)

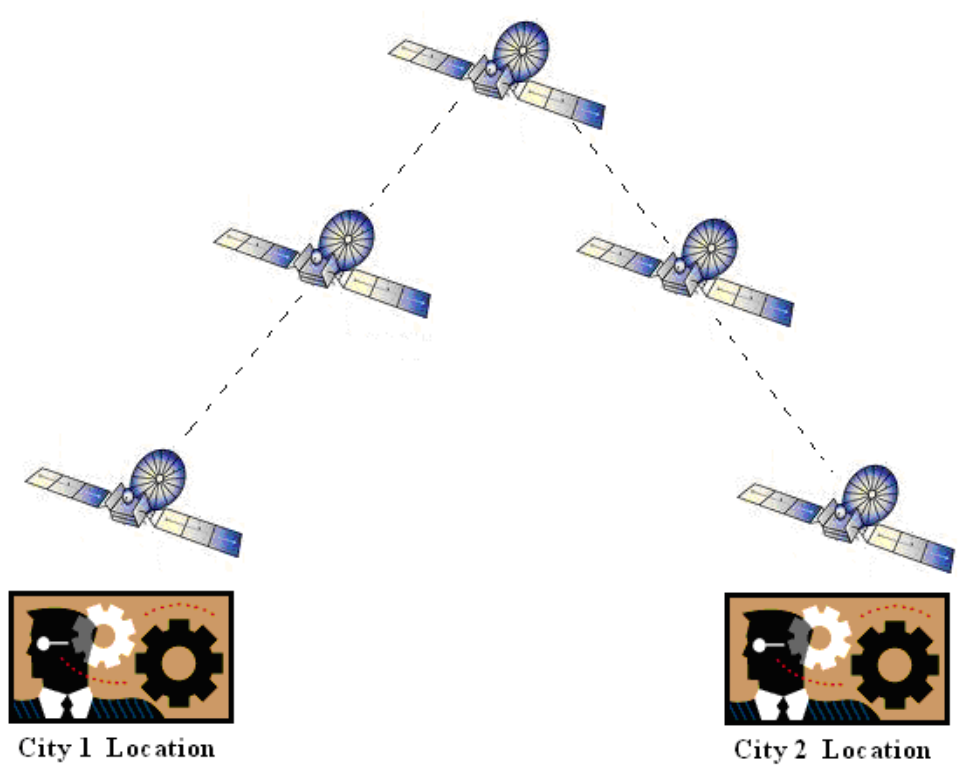

(b)

Figure 3. (a) An example of transmission sketch from city 1: location to city 2: location includes multiple transmission towers; (b) An example of transmission sketch from city 1: location to city 2: location includes multiple transmission satellites

Methodology used in calculating the distances between two cities is for corresponding latencies reported in (Global IP Network, 2009) from the Internet sites such as http://www.yahoo.com and http://www.google.com. Simply, the cities are entered to calculate the distances between US cities. Table 1 provides a partial list of latencies and distances so obtained. A complete list has been used to obtain the scatter graphs necessary, data for the some calculations needed, and verifications to be done. 
Table 1. Network latencies and distances between selected US cities

\begin{tabular}{lccccccc}
\hline & Phoenix & San Antonio & San Diego & San Francisco & St. Louis & Seattle & Washington \\
\hline Atlanta & $41(1591)$ & $28(877)$ & $48(1889)$ & $63(2138)$ & $20(540) \dagger$ & $73(2179)$ & $19(553)$ \\
Austin & $26(867)$ & $12(68)$ & $33(1152)$ & $47(1495)$ & $24(790)$ & $70(1784)$ & $42(1314)$ \\
Cambridge & $70(2293)$ & $57(1755)$ & $78(2577)$ & $78(2694)$ & $34(1160)$ & $74(2485)$ & $11(869)$ \\
Chicago & $51(1449)$ & $37(1041)$ & $51(1729)$ & $51(1855)$ & $10(290)$ & $47(1733)$ & $21(596)$ \\
Cleveland & $51(1748)$ & $37(1248)$ & $58(2032)$ & $57(2167)$ & $14(541)$ & $54(2025)$ & $14(301)$ \\
Dallas & $22(885)$ & $9(243)$ & $29(1181)$ & $44(1480)$ & $20(620)$ & $67(1680)$ & $38(1182)$ \\
Denver & $37(584)$ & $29(799)$ & $30(831)$ & $30(947)$ & $21(831)$ & $53(1017)$ & $42(1491)$ \\
Detroit & $54(1685)$ & $41(1228)$ & $58(1966)$ & $58(2087)$ & $18(542)$ & $54(1931)$ & $17(397)$ \\
Houston & $25(1014)$ & $5(182)$ & $32(1300)$ & $46(1640)$ & $25(853)$ & $69(1889)$ & $44(1220)$ \\
Indiana Polis & $47(1498)$ & $33(995)$ & $54(1786)$ & $56(1947)$ & $6(230)$ & $53(1866)$ & $27(491)$ \\
Kansas City & $36(1042)$ & $22(693)$ & $43(1329)$ & $45(1500)$ & $6(245)$ & $59(1499)$ & $33(946)$ \\
Los Angeles & $11(358)$ & $30(1206)$ & $3(111)$ & $11(342)$ & $53(1812)$ & $35(960)$ & $68(2298)$ \\
Madison & $55(1391)$ & $42(1059)$ & $56(1663)$ & $55(1766)$ & $15(353)$ & $52(1618)$ & $25(706)$ \\
Nashville & $40(1443)$ & $26(813)$ & $47(1740)$ & $62(1960)$ & $12(309)$ & $65(1972)$ & $25(567)$ \\
New Orleans & $32(1313)$ & $13(499)$ & $41(1604)$ & $53(1920)$ & $33(671)$ & $79(2098)$ & $44(966)$ \\
New York & $65(2142)$ & $51(1575)$ & $73(2429)$ & $70(2570)$ & $29(945)$ & $68(2404)$ & $6(206)$ \\
Orlando & $46(1866)$ & $26(1053)$ & $53(2159)$ & $67(2458)$ & $31(986)$ & $84(2565)$ & $30(751)$ \\
Philadelphia & $62(2076)$ & $48(1494)$ & $69(2366)$ & $68(2518)$ & $25(875)$ & $65(2374)$ & $3(120)$ \\
Phoenix & $0(0)$ & $20(849)$ & $7(298)$ & $21(648)$ & $42(1492)$ & $45(1112)$ & $60(1978)$ \\
San Antonio & $20(849)$ & $0(0)$ & $27(1129)$ & $41(1486)$ & $28(897)$ & $64(1783)$ & $46(1377)$ \\
San Diego & $7(298)$ & $27(1129)$ & $0(0)$ & $15(453)$ & $48(1823)$ & $38(1062)$ & $67(2270)$ \\
San Francisco & $21(648)$ & $41(1129)$ & $15(453)$ & $0(0)$ & $50(2014)$ & $24(684)$ & $71(2438)$ \\
St. Louis & $42(1492)$ & $28(897)$ & $48(1823)$ & $50(2014)$ & $0(0)$ & $57(2124)$ & $28(821)$ \\
Seattle & $45(1112)$ & $64(1783)$ & $38(1062)$ & $24(684)$ & $57(2124)$ & $0(0)$ & $68(2324)$ \\
Washington & $60(1978)$ & $46(1377)$ & $67(2270)$ & $71(2438)$ & $28(821)$ & $68(2324)$ & $0(0)$ \\
\hline
\end{tabular}

†numbers in parentheses are the distances rounded to nearest miles for the latencies provided in milliseconds.

The evidence of a linear relationship between distances and latency times is obtained using a scatter diagram of distance (d) vs. latency time (t) and is evident from Figure 4.

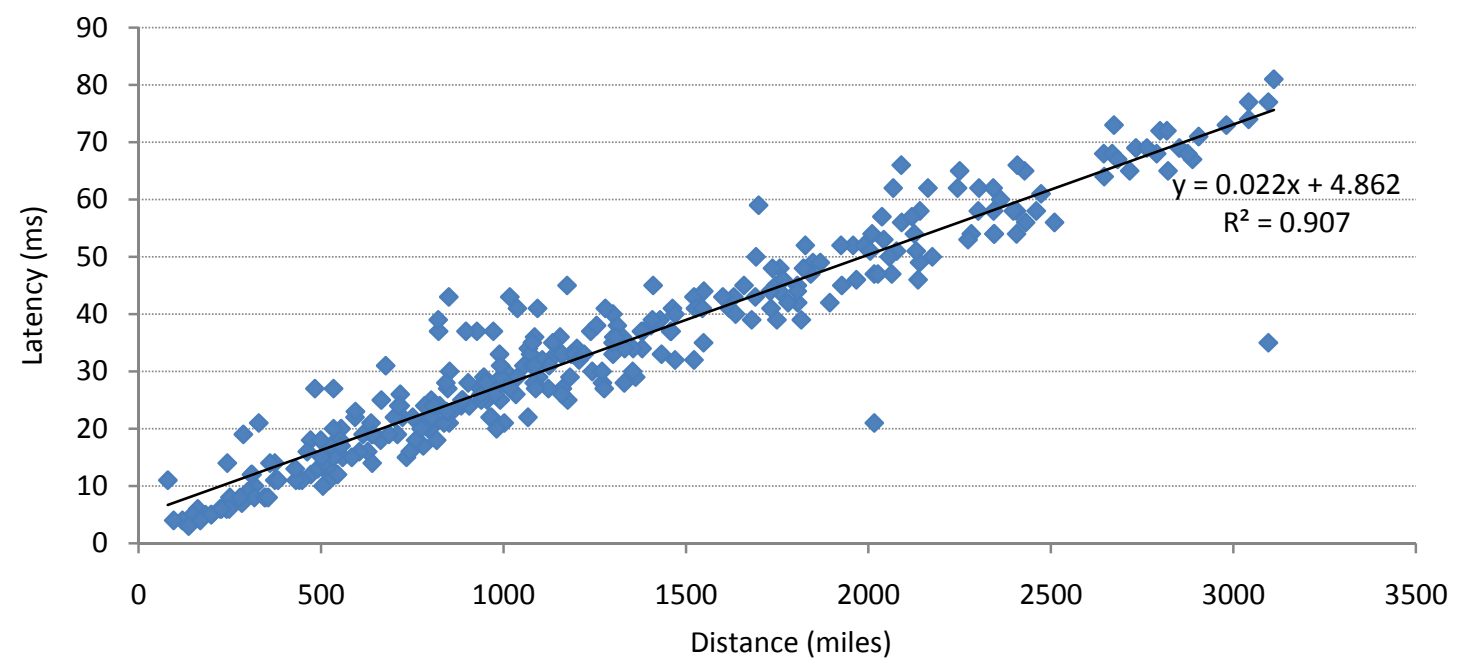

Figure 4. A scatter diagram of distance (d) vs. latency time (t) 
Beyond the latency, other errors are possible in the transmission. For example, if the probability of error in the transmission of a binary digit over a communication is $10^{-3}$, then an expression for the exact probability of more than 3 errors when transmitting a block of $10^{3}$ bits assuming independence of these errors to one another is given by

$$
\operatorname{Pr}\{\text { Errors }>3\}=1-\operatorname{Pr}\{\text { Errors } \leq 3\}=1-\sum_{\mathrm{i}=0}^{3}\left(\begin{array}{c}
1000 \\
\mathrm{i}
\end{array}\right)(0.001)^{\mathrm{i}}(0.999)^{1000-\mathrm{i}}=0.01891
$$

Using the Poisson approximation to the binomial distribution, the approximate value for this probability is

$$
\operatorname{Pr}\{\text { Errors }>3\}=1-\operatorname{Pr}\{\text { Errors } \leq 3\}=1-\mathrm{e}^{-1}-\mathrm{e}^{-1}-\frac{1}{2} \mathrm{e}^{-1}=0.01899
$$

This error appears to be somewhat small; however, the effect on volatile market can be significant. The latency has, somewhat, mixed advantages. For example, it is shown that weapons with less precision are less affected by latencies to suggest that the users might adapt, knowingly or not, to higher latencies by choosing to fight with weapons that cause less precision (Beigbeder et al., 2003). For these situations and others, as well, a numerical method based on the Newton forward-difference formula can provide a technique to calculate the distance between two locations from a known latency between them.

\section{Numerical Methods}

The determination of the divided differences from tabulated data points will provide the basis for Newton forward-difference formula (Faires \& Burden, 1993). The $\Delta$ notation associated with this method follows from the following definition. Given the sequence $\left\{p_{n}\right\}_{n=0}^{\infty}$, the forward difference $\Delta p_{n}$ is $\Delta p_{n}=p_{n+1}-p_{n}$ $\Delta p_{n}=p_{n+1}-p_{n}$, for $n \geq 0$, and the higher powers $\Delta^{k} p_{n}$ are recursively defined by $\Delta^{k} p_{n}=\Delta\left(\Delta^{k-1} p_{n}\right)$, for $k \geq 2$. The zeroth divided difference of the function $f$ with respect to $x_{i}, f\left[x_{i}\right]$, is simply the value of $f$ at $x_{i}$. The remaining divided differences are computed recursively. The first divided difference of $f$ with respect to $x_{i}$ and $x_{i+1}$ is denoted $f\left[x_{i}, x_{i+1}\right]=\frac{f\left[x_{i+1}\right]-f\left[x_{i}\right]}{x_{i+1}-x_{i}}$. After the $(k-1)$ st divided differences $f\left[x_{i}, x_{i+1}, x_{i+2}, \cdots, x_{i+k-1}\right]$ and $f\left[x_{i+1}, x_{i+2}, \cdots, x_{i+k-1}, x_{i+k}\right]$ have been determined, the $k$ th divided difference relative to $x_{i}, x_{i+1}, x_{i+2}, \cdots, x_{i+k-1}, x_{i+k}$ is given by

$$
f\left[x_{i}, x_{i+1}, x_{i+2}, \cdots, x_{i+k-1}, x_{i+k}\right]=\frac{f\left[x_{i+1}, x_{i+2}, \cdots, x_{i+k}\right]-f\left[x_{i}, x_{i+1}, \cdots, x_{i+k-1}\right]}{x_{i+k}-x_{i}}
$$

The Newton forward-difference formula with the notations introduced above is $P_{n}(x)=\sum_{k=0}^{n}\left(\begin{array}{l}s \\ k\end{array}\right) \Delta^{k} f\left(x_{0}\right)$.

Approximating a function using both polynomials and linear regression models is also desired. If the data is accurate and an approximating function that interpolates the given function at the nodes, the interpolating polynomial $P_{n}(x)$ is the polynomial of least degree $n$ that satisfies for a given function $f$ such that $f\left(x_{i}\right)=P_{n}\left(x_{i}\right)$ for $i=0,1,2, \ldots, n$. In order to obtain interpolation polynomial of degree 6 , divided differences are calculated in Table 2 .

Table 2. Divided differences calculated from partial data in the neighborhood of latency of $5 \mathrm{~ms}$

\begin{tabular}{ccccccccc}
\hline $\mathrm{i}$ & $\mathrm{x}_{\mathrm{i}}$ & $\mathrm{f}_{\mathrm{i}}$ & $\Delta^{1}$ & $\Delta^{2}$ & $\Delta^{3}$ & $\Delta^{4}$ & $\Delta^{5}$ & $\Delta^{6}$ \\
\hline 0 & 4 & 169 & 36.00 & -7.88 & 2.54 & -0.25 & -0.04 & 0.02 \\
1 & 6 & 241 & 4.50 & 7.38 & 0.56 & -0.60 & 0.16 & \\
2 & 8 & 250 & 34.00 & 10.75 & -4.25 & 1.00 & & \\
3 & 10 & 318 & 77.00 & -14.75 & 3.73 & & & \\
4 & 12 & 472 & 18.00 & 7.63 & & & & \\
5 & 14 & 508 & 48.50 & & & & & \\
6 & 16 & 605 & & & & & & \\
\hline
\end{tabular}


The coefficients of the Newton forward divided difference form of the interpolatory polynomial are along the first row in the Table 2. The interpolation polynomials of degreed 3, 4, 5, and 6 are derived to calculate the distance that has caused a latency of $5 \mathrm{~ms}$ below.

$$
\begin{aligned}
& P_{3}(5)=169+(5-4) 36+(5-4)(5-6)(-7.88)+(5-4)(5-6)(5-8)(2.54)=220.50 \\
& P_{4}(5)=220.50+(5-4)(5-6)(5-8)(5-10)(-0.25)=224.25 \\
& P_{5}(5)=224.25+(5-4)(5-6)(5-8)(5-10)(5-12)(-0.04)=220.05 \\
& P_{6}(5)=220.05+(5-4)(5-6)(5-8)(5-10)(5-12)(5-14)(0.02)=201.15
\end{aligned}
$$

Among these distances, a distance of 199 miles is reported for latency of $5 \mathrm{~ms}$ providing that the interpolatory polynomial of higher degree will provide better accuracy. However, one should not believe that the degree of the polynomial model can be increased to come up with a perfect model. There is also an optimal degree needed to be identified considering the accumulation of round off errors (including underflow and overflow) (Burden and Faires, 1997). There is a fundamental problem with the polynomial regression models even though several models are described by polynomial equations. This means that the best-fitting results can rarely be interpreted in terms of these models. Polynomial regression can be useful to create a standard curve for interpolation purposes, or to create a smooth curve fitting for graphing. But the polynomial regression is rarely useful for fitting a model to economic data, because a proper layman's interpretation has to be created to convince business communities and the general public (Cramer \& Appelbaum, 1978).

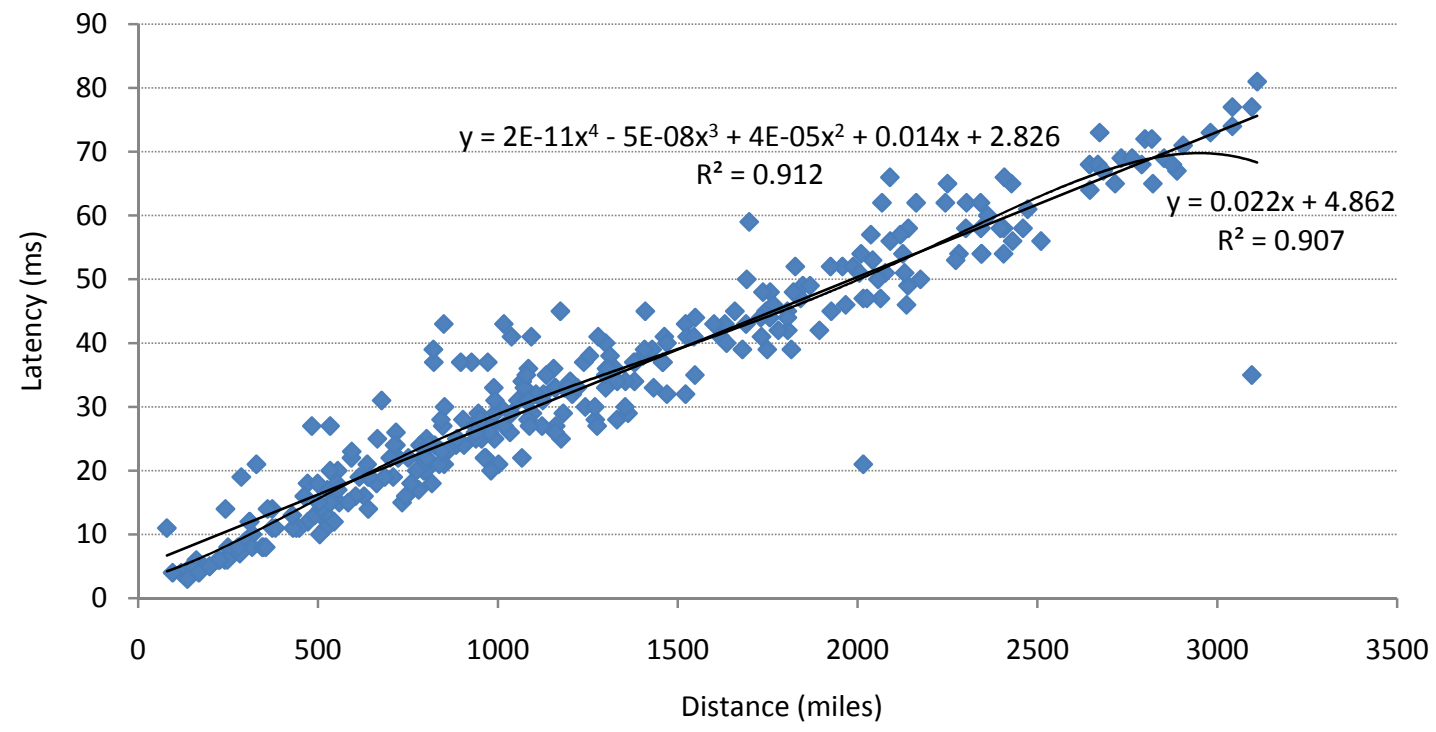

Figure 5. Nonlinear sixth degree polynomial model for latency

Many nonlinear correlation models derived from the data suggested that the sixth degree polynomial provided the highest coefficient of determination $\left(R^{2}=0.9076\right)$ as depicted in Figure 5. From the nonlinear correlation using polynomial of degree 6 providing higher correlation, it is calculated a distance of 117.62 miles. This is far below the actual distance found from the internet cites. It is obvious that the correlation can be increased from choosing polynomial regression of larger degrees may not provide desired results with requiring both higher correlation and higher degrees. So, this may not be the proper direction for our approach as other factors effecting latency cannot be completely ignored.

Reduction of latency is an arduous task. Investigations of factors affecting latency would shed light on how this could be prevented. Modeling latency is primarily done to address the concerns of those who constantly find a way to provide hardware applications to reduce latency. Latency can be detrimental to market conditions. It can lead to loss of clientele and of investment portfolios which will have an impact on the stock market. First of all, through its implication on volatile market conditions and later, on wireless network and packet transmission, this will be discussed further in two sections to follow prior to conducting a reliability analysis to determine the quality of a network as a result of network latency. 


\section{Effect on Volatile Market}

Network latency can play a huge effect on volatile market. Curbing network latency is therefore more important than ever in these turbulent market conditions. As market activities are more volatile, large volumes of traffic can overwhelm systems that could lead to networking and the broader market implications due latency. This could result in either heavy losses or heavy gains in the market. This paper analyzes this situation using five know stocks and implication to the market condition from the latency effect. For example, the network travel time between New York and Chicago is about $7 \mathrm{~ms}$ and Tokyo and London is about $120 \mathrm{~ms}$ whereas a trader needs to take trading decision instantaneously resulting in an effect on market condition (Sinclair, 2008). These models can retrace the actual price prevailing at that time simultaneously to make appropriate decision on trading from the existing data using facsimile transmission or e-mail communication. The findings of this study have important implications for both scholarly work and for business communities. Firstly, the results of the stock market's performance are naturally the reflection of someone's personal wealth. However, a small portion of sums exchanged each day on the world's stock markets through companies depends on their financial needs and prospects. Actually, pre-programmed computers carry out necessary recognition of their own stock prices and arrange investments almost instantaneously. The investing components of these transactions are mostly mathematics using hyper-speed electronic equipment (Toffler \& Toffler, 2006).

\section{Latency on Wireless Network and Packet Transmission}

Latency on the other forms of network communication is somewhat beyond the distance between them. Packets move the progression of communication from one end to another. So, the discussions on wireless network and packet transmission are worth being noted. One of the most important drawbacks in the quality of wireless communication has been latency. It is desirable to have low latency in order to have high quality transmission. Wireless Local Area Network (WLAN) systems usually experience higher delay when compared with that of a wired LAN. This can be due to the presence of more network traffic and loss of packets during the transmission. In the case of WLAN, if the number of users is increased, then the quality of the communication deteriorates quickly. If they try to obtain the same access point then there will be more network jitters, especially, when the large packets are sent across the network in the same direction. Also, the fast handoff mechanism does not require any special radio technology features, modifying routing mechanism in the wired network, or supports of specific technology (Shim et al., 2002).

Latency and other factors in VoIP (Voice over Internet Protocol) over WLANs are important to consider. The VoIP is one of the areas that is being increasingly used today, furthermore, due to the improved and increased WLAN the VoIP is being implemented over the WLANs to avoid jittering. In addition, more and more television networks and Internet sites use streaming video for real-time broadcasting. At times, the reception quality is not as good as one would expect. Evaluation of packet loss impairment on streaming video has been well-studied and would not provide glooming picture, as well (Rui, Li, \& Qiu, 2006). However, the urgency need of the new items to be broadcast the quality has put into a backburner. Through a combination of techniques, it is possible to deliver high quality and interactive videos in real-time (Feamster \& Balakrishnan, 2002). The increasing use of commercial off-the-shell (COTS) hardware and software products possess a greater risk as wireless components are commonly found in these product tools (Longstaff, Haimes, \& Sledge, 2002). The associated tradeoffs in terms of costs, benefits, and risks need to be analyzed as the industries' use of these products is on the rise.

Bandwidth represents the overall capacity of the network connection. If the capacity is greater, then it is more likely that the network will perform better. Bandwidth is the amount of data that passes through a network connection over time as measured in bps (bits per second). If we take a communications wireless network as an example for explaining the path then the data packet travel can be depicted using source, intermediate, and destination satellites. At the same time, the path in which the data packets travel should always be as short as possible in order to reduce the latency, high-system efficiency and to maintain the synchronization between the data.

It is desirable to know how the packet travels in a network in packet communication, how it is identified, and how it is sent to its destination. The packet travels in the network once the IP address has been determined. The packets, then travel through many intermediate machines or nodes which can include routers, bridges, etc. Once the packet arrives to the router, the machine checks the header and decides where to send the packet (Goh, Kahng, \& Kim, 2003). If the address mentioned in the header matches with the machine, then the packet is kept and passed to the higher level protocol. If the address does not match the descriptions it is passed to another machine before passing it onto another machine. The machine searches for its Routing Information Table (RIT) to know other corresponding details. For example, dynamic RIT is used to efficiently identify the destination of 
the packets. If a packet has to travel from one destination to another it can travel in many ways including paths and networks.

\section{Reliabilities}

Reliability is a measure of continuous and uninterrupted delivery of quality service to make a system work as expected (Laprie, 1985). Blocks represent system components. Lines describe the connections between them. A Reliability Block Diagrams (RBD) is a graphical depiction of the system's components and connectors which can be used to depict the functional of the system and to determine the overall system reliability. If any path through the system is successful, then the system would succeed, otherwise it fails to function. Several assumptions are needed first. Lines assume to have reliability equals to 1. Failures of blocks are statistically independent and blocks represent one of the two possibilities from a total failure to working completely.

Latency can definitely cause accumulated risk as is evident from the several discussions made earlier. It is; therefore, appropriate to begin reliability analysis for a network consisted of parallel and series components. A summation system of $n$ units with system reliability

$$
p(x, s)=\sum_{i=1}^{n} p_{i}\left(x_{i}, s_{i}\right), \quad p_{i}\left(x_{i}, s_{i}\right)=\left\{\begin{array}{l}
1 / n \text { if } s_{i}=1 \\
0 \quad \text { if } \mathrm{s}_{\mathrm{i}}=0
\end{array}, \quad x=\left(x_{1}, x_{2}, \ldots, x_{n}\right) \text { and } s=\left(s_{1}, s_{2}, \ldots, s_{n}\right)\right.
$$

where $p_{i}\left(x_{i}, s_{i}\right)$ is conveniently defined to be equal for all components, functions additively more reliable as long as the more reliability each component of the network.

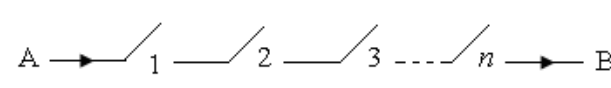

A Series System of $n$ Units

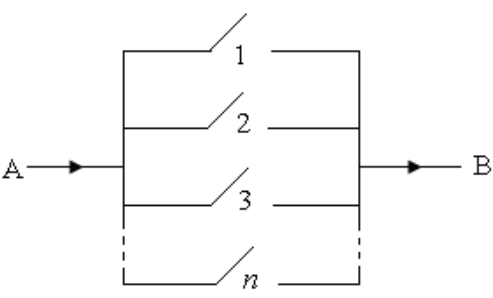

A Parallel System of $n$ Units

Figure 6. A Series system of $n$ units and a parallel system of $n$ units

A series system of $n$ units with system reliability $p(x, s)=\prod_{i=1}^{n} p_{i}\left(x_{i}, s_{i}\right), x=\left(x_{i}, x_{2}, \ldots, x_{n}\right)$, and $s=\left(s_{1}, s_{2}, \ldots, s_{n}\right)$ functions if each of the units functions and a parallel system of $n$ units with system reliability $p(x, s)=1-\prod_{i=1}^{n}\left[1-p_{i}\left(x_{i}, s_{i}\right)\right], x=\left(x_{i}, x_{2}, \ldots, x_{n}\right)$, and $s=\left(s_{1}, s_{2}, \ldots, s_{n}\right)$, functions if at least one of units functions.

Estimating vulnerability risk through Probabilistic Risk Analysis (PRA) is primarily a mathematical approach to assessing the reliability of the network system, containing either in series or parallel components, or both. The goal of PRA is to merge reliability with the network to produce more accurate estimates of the system reliability (Hausken, 2002). This matters because risk is determined both by the most vulnerable components of the system and the controllable risk.

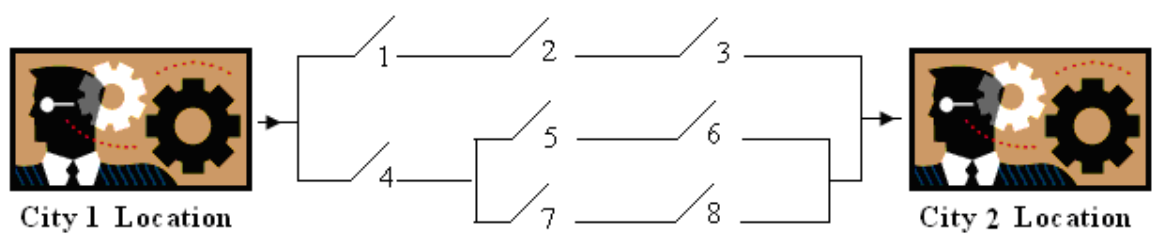

Figure 7. Schematic diagram consists of combined parallel and series components

The reliability block diagram in Figure 7 consists of eight combined series and parallel components and has system reliability; $R=1-\left(1-p_{1} p_{2} p_{3}\right)\left[1-p_{4}\left[1-\left(1-p_{5} p_{7}\right)\left(1-p_{6} p_{8}\right)\right]\right]$. Reliability of any other schematic diagram can be calculated similarly. A scheme equivalent to Dijkstra's algorithm (Appleby \& Steward, 2000; 
Ahuja, Magnanti, \& Orlin, 1993; Johnson, 1973; Zhan, 1997; Orda \& Rom, 1990) can evaluate the minimum probability between the entry to the network system and until the time that a network component is reached to another user. In order to express reliability, it is assumed that a homogeneous failure rate $\lambda$ (per unit of time $t$ ) constitutes reliability over a period $T$ of $R=e^{-\lambda T / t}$. For example, if a component fails at the rate of 10 failures per 1000 hours, then the reliability over a 30 hour period is approximately $74 \%$.

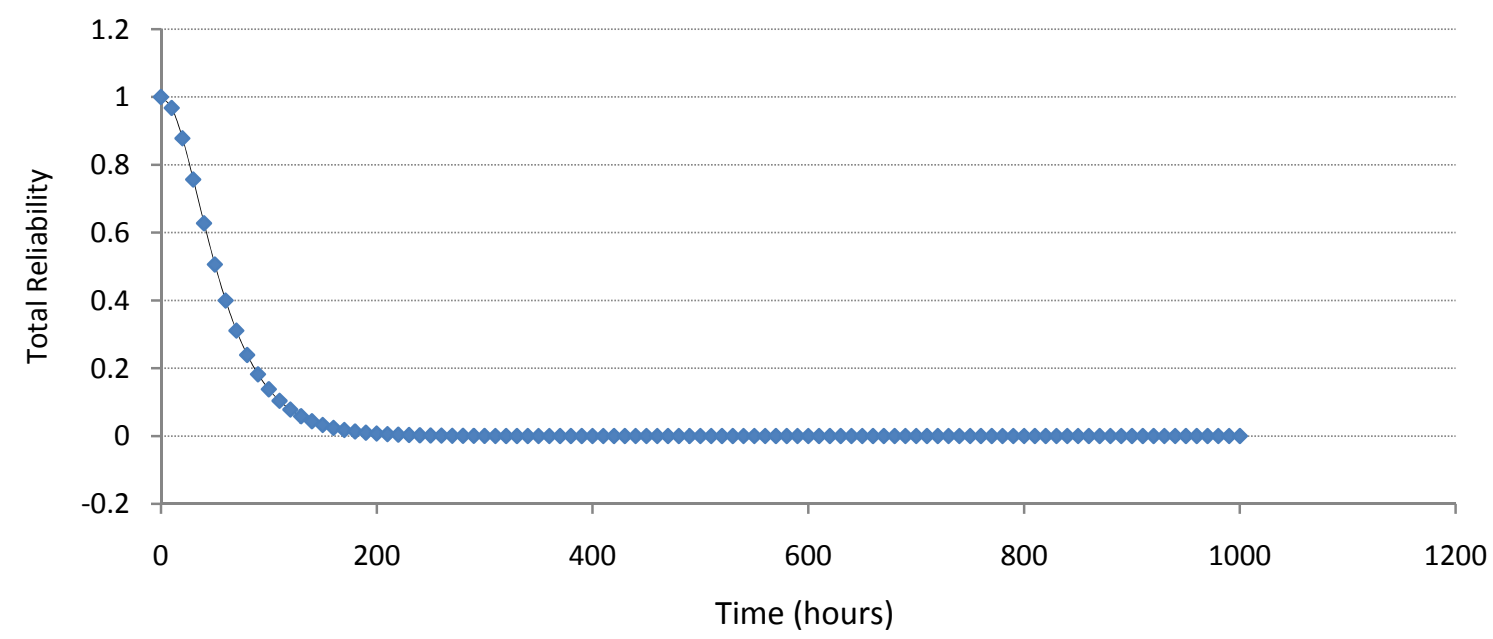

Figure 8. Reliability chart schematic diagram

Figure 8 depicts possible system reliability over an extended time period assuming a component failure rate of 10 failures per 1000 hours and all eight components have the same rate of failure.

\section{Conclusions}

In this paper, presented are some statistical and numerical methods of estimating latency and how it helps in the determination of number of satellites used in the transmission from a linear regression model. Numerical methods based on divided-difference are introduced to generate successively the higher-degree polynomials themselves to determine the actual latencies occurred. These models also provide evidence of location; from where the communications have originated. However, when calculating the distances between cities, they can be differed from 0-40 miles, at least, since a city is a large geographical area. This may have resulted in some verifications being incorrect, as actual exact locations used for calculating the latencies provided in the data are unknown. Analysis demonstrated that the system reliability deteriorates over the time of transmission.

\section{Acknowledgements}

The authors would like to thank two reviewers whose comments and insights vastly assisted to improve this article.

\section{References}

Ahuja, R. K., Magnanti, T. L., \& Orlin, J. B. (1993). Network Flows: Theory, Algorithms, and Applications. Prentice Hall.

Anant, A. (1991). Limits on Interconnection Network Performance. IEEE Transactions on Parallel and Distributed Systems, 2(4), 398-412. http://dx.doi.org/10.1109/71.97897

Appleby, S., \& Steward, S. (2000). Mobile Software Agents for Control in Telecommunications Networks. BT Technology Journal, 18(1), 68-70. http://dx.doi.org/10.1023/A:1026569720098

Ariel, O., \& Raphael, R. (1990). Shortest-Path and Minimum-Delay Algorithms in Networks with Time-Dependent Edge-Length. Journal of the ACM (JACM), 37(3), 607-625. http://dx.doi.org/10.1145/79147.214078

Beigbeder, T., Coughlan, R., Lusher, C., Plunkett, J., Agu, E., \& Claypool, M. (2003). The Effects of Loss and Latency on User Performance in Unreal Tournament. SIGCOMM'04 Workshops, Aug. 30-Sept. 3, 2004, Portland, Oregon, pp. 144-151.

Donald, B. J. (1973). A Note on Dijkstra's Shortest Path Algorithm. Journal of the ACM (JACM) archive, 20(3), 385-388. 
Douglas, J. F., \& Richard, L. B. (1993). Numerical Methods. PWS Publishing Company.

Elliot, M., Cramer, \& Mark, I. A. (1978). The Validity of Polynomial Regression in the Random Regression Model. Review of Educational Research, 48(4), 511-515.

Fujiwhara, S. (1916). On The Abnormal Propagation of Sound Waves in the Atmosphere, Second Part, Bull. Central Met'l Obs'y, Tokyo, Japan, 2, pt. 4, pl., 82, p. 27 lith, $4^{\circ}$.

Global IP Network. (2009) Retrieved March 3, 2009, from http://ipnetwork.bgtmo.ip.att.net/pws/netwrok_delay.html

Goh, K. I., Kahng, B., \& Kim, D. (2003). Packet Transport and Load Distribution in Scale-Free Network Models. Physica A: Statistical Mechanics and its Applications, 318(1-2), 72-79.

Harsha, V. M., Thomas, A., Arvind, K., Neil, S., \& Arun, V. (2006). A Structural Approach to Latency Predication, IMC'06, October 25-27, Rio de Janeiro, Brazil, pp. 99-104.

James, F. K., \& Keith, W. R. (2012). Computer Networking: A Top-Down Approach (6th ed.). Pearson Education, Inc.

Jena-Claude, L. (1985). Dependable Computing and Fault Tolerance: Concepts and Terminology. In Proceedings of the 15th International Symposium on Fault-Tolerant Computing (FTCS-15), pp. 2-11.

Jesper, M. J. (2000). On the Impact of Network Latency on Distributed Systems Design. Information Technology and Management, 1(3), 183-194. http://dx.doi.org/10.1023/A:1019121024410

Kjell, H. (2002). Probabilistic Risk Analysis and Game Theory. Risk Analysis, An International Journal, 22(1), $17-27$.

Nick, F., \& Hari, B. (2002). Packet Loss Recovery for Streaming Video. Proceedings of the 12th International Packet Video Workshop.

Richard, L., Burden, \& Douglas, J. F. (1997). Numerical Analysis (6th ed.). Pacific Grove, CA: Brooks/Cole Publishing Company.

Rui, H., Li, C., \& Qiu, S. (2006). Evaluation of Packet Loss Impairment on Streaming Video. J Zhejiang Univ SCIENCE B, 1, 1-7.

Shim, E., Wei, H. Y., Chang, Y., \& Gitlin, R. D. (2002). Low Latency Handoff IP QOS with NeighborCasting. IEEE, pp. 3245-3249.

Sinclair, J. (2008). Distance Latency: Overcoming the Constraints of Geography in FX, Algorithmic FX Trading, e-FOREX.

Sinha, P., Raz, D., \& Choudhuri, N. (2006). Estimation of Network Distances Using Off-Line Measurements. Computer Communications, 29, 3295-3305. http://dx.doi.org/10.1016/j.comcom.2006.05.010

Ström, G. E., Parkvall, S., \& Ottersten, E. B. (1994). Near-Far Resistant Propagation Delay Estimators for Asynchronous Direct-Sequence Code Division Multiple Access Systems. Proceedings of the 1994 International Zurich Seminar on Digital Communications: Mobile Communications: Advanced Systems and Components, pp. 251-260, Springer-Verlag London.

Thomas, A. L., Yacov, Y. H., \& Carol, S. (2002). Are We Forgetting the Risk of COTS Products in Wireless Communications? Risk Analysis, An International Journal, 22(1), 1-6.

Toffler, A., \& Toffler, H. (2006). Revolutionary Wealth. New York: Alfred A. Knopf.

Wolman, A., Voelker, G., \& Thekkath, A. C. (1994). Latency Analysis of TCP on an ATM Network. Proceedings of the 1994 USENIX Winter Technical Conference, San Francisco, CA, USA, pp. 167-179.

Zhan, B. F. (1997). Three Fastest Shortest Path Algorithms on Real Road Networks: Data Structures and Procedures. Journal of Geographic Information and Decision Analysis, 1(1), 69-82. 